\title{
APLICAÇÃO DA ESPECTROSCOPIA RAMAN NA CARACTERIZAÇÃO DE MINERAIS PERTENCENTES A UMA GEOCOLEÇÃO
}

\author{
Adriele R. Santos ${ }^{\mathrm{a}, \mathrm{b}}$, Denise B. Menezes ${ }^{\mathrm{c}}$, Javier Ellena ${ }^{\mathrm{a}, *,(1)}$ e Marcelo B. Andrade ${ }^{\mathrm{a}}$ \\ 'Instituto de Física de São Carlos, Universidade de São Paulo, 13566-590 São Carlos - SP, Brasil \\ bDepartamento de Química, Universidade Federal de São Carlos, 13565-905 São Carlos - SP, Brasil \\ 'Departamento de Engenharia Civil, Universidade Federal de São Carlos, 13565-905 São Carlos - SP, Brasil
}

Recebido em 31/01/2019; aceito em 26/03/2019; publicado na web em 18/04/2019

\begin{abstract}
RAMAN SPECTROSCOPY APPLICATION IN THE CHARACTERIZATION OF PERTAINING MINERALS OF A GEOCOLLECTION. Lots of Educational Institutions hold their own geocollection due to its utility and versatileness in the practical formation of new generations of skillful professionals in many areas of Science and Technology. The Geoscience laboratory of the Federal University of São Carlos has a collection of minerals used in engineering, mineralogy and other technological courses ministered to undergraduates of different programs. Three samples of this collection were identified using Raman spectroscopy. The analysis carried out in this work enabled the characterization of the major mineral components in the samples as well as the identification of other minor crystalline phases present in the sample. It is important to notice that this information could not be obtained using the regular analyses of mineral properties, such as density, streak, hardness, brightness, cleavage, among others. As a result, the samples A170801 and A170802 were characterized as being mainly calcite $\left(\mathrm{CaCO}_{3}\right)$ and spodumene $\left(\mathrm{LiAlSi}_{2} \mathrm{O}_{6}\right)$, respectively. On the other hand, sample $\mathrm{A} 170803$ show the presence of three different minerals: graphite $(\mathrm{C})$, quartz $\left(\mathrm{SiO}_{2}\right)$ and muscovite $\left[\mathrm{KAl}_{2}\left(\mathrm{Si}_{3} \mathrm{Al}\right) \mathrm{O}_{10}(\mathrm{OH}, \mathrm{F})_{2}\right]$.
\end{abstract}

Keywords: geocollection; Raman spectroscopy; mineralogy.

\section{INTRODUÇÃO}

Os minerais sempre desempenharam um papel importante no modo de vida da humanidade. Na pré-história, a hematita $\left(\mathrm{Fe}_{2} \mathrm{O}_{3}\right)$, a goethita $(\alpha-\mathrm{FeOOH})$ e os óxidos de manganês eram empregados como pigmentos nas pinturas rupestres. ${ }^{1-4}$ Minerais também foram utilizados na antiguidade pelas civilizações assíria, egípcia, babilônica, grega e romana, como insumos medicinais. ${ }^{5}$ Com o passar dos tempos, os minerais tornaram-se cada vez mais relevantes e recorrentes na vida do homem e, hodiernamente, possuem elevada importância devido à profusão de significados e empregabilidade que apresentam.

Eles são a principal fonte de metais, produtos químicos inorgânicos e matérias-primas essenciais ao desenvolvimento das indústrias farmacêutica, de fabricação, construção civil, petróleo e de alta tecnologia. No Brasil, por exemplo, o espodumênio, principal fonte de lítio do país, é beneficiado para a produção de hidróxidos e carbonatos desse elemento químico, que são empregados na confecção de baterias automotivas, vidros, cerâmicas e lubrificantes. ${ }^{6}$ Outro mineral importante é a calcita, principal constituinte de calcários de alta pureza, que é utilizada na construção civil e na otimização das propriedades físicas de cerâmicas, adesivos e tintas. ${ }^{7}$

Tendo em vista a importância desses recursos geológicos como fontes de matéria prima para o fomento de diversos setores industriais e para o entendimento da história e estrutura da Terra, ${ }^{8}$ há uma necessidade de formar técnicos e investigadores com conhecimento em geociências e mineralogia. Neste sentido, as geocoleções têm se mostrado como uma importante ferramenta na formação prática de novas gerações de profissionais qualificados em muitos campos da Ciência e Tecnologia. ${ }^{9}$

As coleções de minerais representam um recurso cultural relevante para a difusão do conhecimento científico e tecnológico, ${ }^{10}$ dado que elas possuem diversas finalidades, tais como pesquisa,

*e-mail: javiere@ifsc.usp.br exposição permanente ou mesmo usos educacionais e didáticos. ${ }^{11}$ Em virtude disso, várias instituições ligadas às ciências geológicas detêm um acervo mineralógico. O Departamento de Engenharia Civil da Universidade Federal de São Carlos (UFSCar) possui uma coleção mineralógica que é empregada em disciplinas ministradas aos alunos de graduação de diferentes cursos da universidade. Desse modo, a correta identificação de seus minerais se faz necessária, pois devido a sua importância, as coleções devem ser preservadas e periodicamente revistas e analisadas. ${ }^{11}$

Considerando o panorama apresentado, este trabalho tem como objetivo caracterizar três amostras pertencentes à coleção mineralógica do Departamento de Engenharia Civil da UFSCar. Com base nesse objetivo será empregada a Espectroscopia Raman, uma vez que essa se configura como uma técnica rápida e eficiente para a identificação de minerais, além de fornecer informações que não podem ser obtidas por meio da análise das propriedades comuns de caracterização como densidade, traço, dureza, brilho, clivagem, dentre outras. Assim sendo, os resultados oriundos deste trabalho poderão ser empregados em atividades de pesquisa, disciplinas acadêmicas e exposições didáticas.

\section{Espectroscopia Raman}

As técnicas espectroscópicas são constantemente utilizadas na identificação, caracterização e elucidação de estruturas de moléculas e compostos, bem como no monitoramento e controle de reações químicas. Elas estão fundamentadas na propriedade que átomos e moléculas têm de absorver ou emitir energia em uma determinada região do espectro eletromagnético, o que oferece informações sobre a composição química e estrutura cristalina da amostra. ${ }^{12}$

Ao incidir radiação sobre um corpo, pode haver uma transição entre seus estados energéticos e, dependendo da região em que a energia da radiação incidente se encontra, ocorrem tipos distintos de transições como as eletrônicas, que estão comumente estabelecidas na região do ultravioleta ou visível, as rotacionais, definidas na região de 
micro-ondas, as translacionais e as vibracionais, essas últimas situadas no visível, infravermelho ou infravermelho próximo. ${ }^{13}$

As transições vibracionais são estudadas através da espectroscopia vibracional que abrange três tipos de técnicas analíticas: a espectroscopia de absorção no infravermelho, espectroscopia Raman (espalhamento Raman) e a espectroscopia de espalhamento de nêutrons. Entretanto, as espectroscopias de infravermelho e a Raman são as mais utilizadas. ${ }^{14}$ Ambas as técnicas medem os níveis de energia vibratória associados às ligações químicas na amostra através da interação da radiação eletromagnética com o movimento vibracional dos núcleos, o que gera o espectro vibracional. ${ }^{13}$ Tais espectros exibem uma maior riqueza de detalhes sobre o material analisado em detrimento dos espectros eletrônicos, que são constituídos por bandas largas e deformadas, enquanto os vibracionais são espectros únicos de cada material e das moléculas que o constituem. ${ }^{15}$

A espectroscopia Raman é uma técnica de espalhamento baseada no Efeito Raman, descoberto e publicado em 1928 pelo físico indiano Chandrasekhara Venkata Raman. O efeito caracteriza-se por uma alteração na frequência de uma pequena fração da radiação incidente quando esta é espalhada por moléculas ou estruturas cristalinas.

Em uma descrição abreviada, o espalhamento ocorre através da interação das moléculas de uma amostra com uma luz monocromática eletromagnética. Nessa interação, os fótons incidentes excitam a molécula que está inicialmente em um nível vibracional do estado eletrônico fundamental para um estado virtual (intermediário), do qual ela se recupera imediatamente (cerca de 10 a $14 \mathrm{~s}$ ), ${ }^{16}$ de três formas distintas.

Na primeira forma (Figura 1, esquema a), o espalhamento ocorre elasticamente (dispersão Rayleigh), uma vez que se emprega o princípio da conservação de energia, pois o fóton é espalhado sem sofrer alteração em seu valor de comprimento de onda, ou seja, a energia do fóton é a mesma antes e após sua interação com a matéria. ${ }^{17}$ Nas outras duas, há a ocorrência de espalhamento inelástico (efeito Raman), que acontece quando a molécula, ao retornar do estado virtual ao estado fundamental, adquire um nível energético mais alto ou mais baixo quando comparado com seu nível inicial. Se a molécula receber energia através da sua interação com o fóton incidente, $\mathrm{E}=$ $h \boldsymbol{v}_{0}$, o fóton espalhado, $\mathrm{E}=h\left(\boldsymbol{v}_{0}-\boldsymbol{v}_{1}\right)$, perderá a mesma porção de energia que a molécula adquiriu. Nesse processo, o fóton espalhado possui energia menor que a do incidente. Esse espalhamento é do tipo Raman Stokes (Figura 1, esquema b).

Entretanto, devido à distribuição de Boltzman $\left(\mathrm{N} / \mathrm{N}_{0},=e^{-(\Delta \mathrm{E} / \mathrm{kT})}\right.$, em que $\mathrm{N}$ é o número de moléculas inicialmente no estado excitado, $\mathrm{N}_{0}$ corresponde ao número de moléculas inicialmente no estado fundamental de vibração e $\Delta \mathrm{E}$ é a diferença de energia entre esses dois estados ${ }^{15}$ é possível que, em condições ambientes, um número finito de moléculas já estejam vibracionalmente excitadas e, ao interagir com a radiação eletromagnética, elas acabem perdendo energia. Neste caso, o fóton espalhado adquire a energia dissipada pelas moléculas,
$\mathrm{E}=h\left(\boldsymbol{v}_{0}-\boldsymbol{v}_{1}\right),{ }^{18}$ o que é caracterizado como espalhamento do tipo Raman anti-Stokes (Figura 1, esquema c).

Tendo em vista o potencial da espectroscopia Raman e os avanços no desenvolvimento de fontes lasers e detectores de maior sensibilidade, essa ferramenta tem sido frequentemente utilizada para fins mineralógicos, o que é amplamente destacado na literatura. ${ }^{2,18-25}$ Ela é comumente empregada na identificação de diversos minerais e pedras preciosas, pois fornece informações sobre a composição molecular, a estrutura e as interações intermoleculares da amostra. ${ }^{18-20,26-29}$ Trata-se de uma técnica não destrutiva e não invasiva e que requer um preparo mínimo da amostra, ${ }^{20,29,30}$ além de possibilitar o estudo de regiões micrométricas e das diferentes fases minerais presentes. Ademais, os espectros Raman apresentam alta resolução ${ }^{31}$ e são como uma "impressão digital" do material analisado, ${ }^{29}$ concedendo detalhes estruturais que possibilitam a identificação de um determinado mineral e de outros materiais sólidos. ${ }^{18,20}$

\section{PARTE EXPERIMENTAL}

\section{Minerais}

Três amostras pertencentes à coleção mineralógica do Departamento de Engenharia Civil da Universidade Federal de São Carlos foram selecionadas para este estudo. Elas foram caracterizadas por espectroscopia Raman sem qualquer tipo de preparo prévio ou manipulação especial.

\section{Espectroscopia Raman}

As medidas de espectroscopia Raman deste trabalho foram realizadas no Instituto de Física de São Carlos (IFSC-USP) no espectrômetro Raman modelo LabRAM HR Evolution da marca Horiba Scientific, equipado com um monocromador Czerny-Turner, um detector CCD e um microscópio confocal Olympus (objetiva de 50x), que é usado para focalizar o laser no ponto desejado da amostra e para detectar a radiação espalhada.

Foram empregadas duas linhas de excitação laser: 532 e 633 nm, cujo critério de seleção consistiu em minimizar efeitos indesejáveis de fluorescência que poderiam prejudicar a identificação das bandas características Raman. A potência do laser na superfície das amostras foi de, aproximadamente, $1 \mathrm{~mW}$ para evitar possíveis efeitos de degradação. Uma grade de difração com densidade de 600 linhas/mm e resolução de $2 \mathrm{~cm}^{-1}$ também foi utilizada (Tabela 1). O software LabSpec6 (Horiba Scientific) foi empregado na operação do equipamento e na aquisição dos espectros. Todos os espectros Raman experimentais foram comparados com a base de dados RRUFF (http:// rruff.info/) e com a literatura, com o objetivo de identificar a composição dos minerais estudados. $\mathrm{O}$ ajuste da linha de base, a remoção

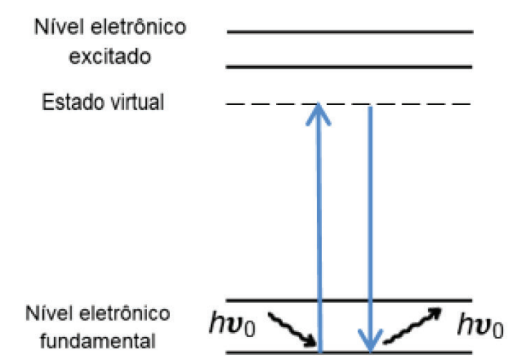

(a)

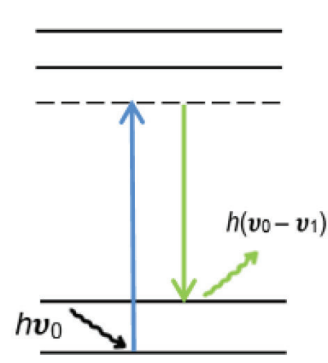

(b)

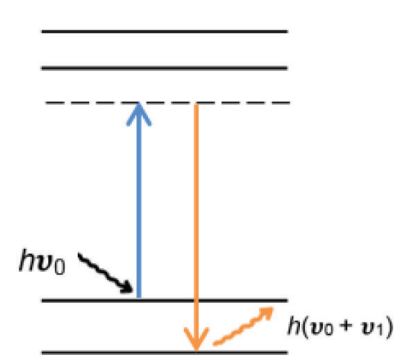

(c)

Figura 1. Espalhamento da radiação eletromagnética: (a) espalhamento elástico (Rayleigh), (b) espalhamento inelástico (região Stokes) e (c) espalhamento inelástico (região anti Stokes) 
Tabela 1. Parâmetros utilizados para a coleta dos espectros Raman das amostras

\begin{tabular}{lcccccc}
\hline Amostra & $\begin{array}{c}\text { Número de } \\
\text { Acumulação }\end{array}$ & $\begin{array}{c}\text { Tempo de aquisição } \\
(\mathrm{s})\end{array}$ & $\begin{array}{c}\text { Linha de excitação } \\
(\mathrm{nm})\end{array}$ & $\begin{array}{c}\text { Densidade da grade } \\
(\text { linhas/ mm })\end{array}$ & $\begin{array}{c}\text { Faixa analisada } \\
\left(\mathrm{cm}^{-1}\right)\end{array}$ & $\begin{array}{c}\text { Potência do laser } \\
(\mathrm{mW})\end{array}$ \\
\hline A170801 & 3 & 10 & 532 & 600 & $100-1400$ & 1 \\
A170802 & 3 & 10 & 633 & 600 & $100-1400$ & 1 \\
A170803a & 3 & 10 & 532 & 600 & $100-1650$ & 1 \\
A170803b & 3 & 20 & 633 & 600 & $100-1800$ & 1 \\
A170803c & 3 & 20 & 633 & 600 & $100-1650$ & 1 \\
\hline
\end{tabular}

do background e a normalização dos espectros ocorreu por meio do software CrystalSleuth, disponível gratuitamente pelo projeto RRUFF.

\section{RESULTADOS E DISCUSSÃO}

As amostras A170801, A170802 e A170803 (Figuras 3, 5 e 7) foram analisadas por espectroscopia Raman, buscando não apenas a caracterização do mineral majoritário que as constituía, mas também a identificação da presença de outras espécies de minerais que compunham tais amostras. Os espectros apresentados a seguir são representativos das amostras supracitadas e as frequências de vibração neles encontrados foram discutidas de acordo com a literatura.

\section{Espectroscopia Raman}

\section{Amostra A170801}

O espectro Raman obtido para a amostra A170801 (Figura 2) é característico do mineral calcita (Figura 3). Ela é uma das formas polimórficas do carbonato de cálcio $\left(\mathrm{CaCO}_{3}\right)$ na natureza, sendo o mineral carbonático mais importante e abundante..$^{32,33}$

Os espectros Raman da calcita são comumente divididos em três seções ${ }^{32}$ sendo elas a região de $1700-1200 \mathrm{~cm}^{-1}$ relacionada com os modos de estiramento assimétrico das ligações $\mathrm{C}-\mathrm{O}$ do grupo $\mathrm{CO}_{3}^{-2}$, a região de $1200-600 \mathrm{~cm}^{-1}$, que é característica ao estiramento simétrico $\mathrm{C}-\mathrm{O}$ do $\mathrm{CO}_{3}^{-2}$ e a região $500-100 \mathrm{~cm}^{-1}$, atribuída às vibrações externas do $\mathrm{CO}_{3}^{-2}$. Analisando-se a Figura 3, é possível observar a presença de quatro bandas bem definidas que foram comparadas com dados presentes na literatura (Tabela 2).

Examinando atentamente a faixa de $1200-600 \mathrm{~cm}^{-1}$ no espectro medido, é notória a presença de uma banda intensa em $1085 \mathrm{~cm}^{-1}$ referente à vibração de estiramento simétrico do grupo carbonato. ${ }^{35}$ De acordo com Frost e colaboradores ${ }^{35}$ a posição desta banda deriva da estrutura cristalina do mineral carbonático. Corroborando com isso, Faria ${ }^{4}$ esclarece que o carbonato de cálcio é encontrado na natureza em três formas cristalinas: calcita, aragonita e vaterita, as quais podem ser diferenciadas por espectroscopia Raman porque, apesar de terem a mesma composição, possuem arranjos cristalinos

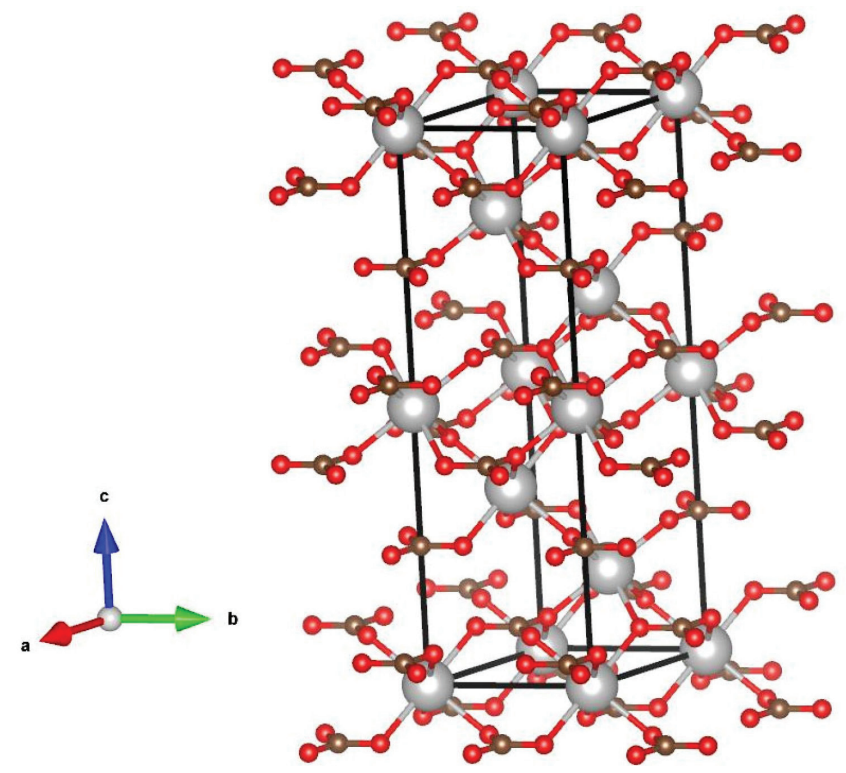

Figura 2. Estrutura cristalina da calcita, $\mathrm{CaCO}_{3}$ : Ca em cinza, $\mathrm{C}$ em marrom e O em vermelho. ${ }^{34}$

Tabela 2. Frequências Raman do mineral calcita $\left(\mathrm{cm}^{-1}\right)$

\begin{tabular}{cccc}
\hline Amostra A170801 & Ref. $^{38}$ & Ref. $^{39}$ & Ref. $^{32}$ \\
\hline 152 & 154 & 155 & 158 \\
279 & 283 & 281 & 287 \\
709 & 714 & 711 & 715 \\
1085 & 1087 & 1085 & 1088 \\
\hline
\end{tabular}

distintos. Ainda, a deformação angular no plano do íon $\mathrm{CO}_{3}{ }^{2-}$ origina uma banda Raman de baixa intensidade em $709 \mathrm{~cm}^{-1}$, sendo esta considerada uma banda característica da calcita. ${ }^{32,36} \mathrm{Na}$ região do espectro Raman de 500-100 $\mathrm{cm}^{-1}$, são observadas duas bandas nos comprimentos de onda, 152 e $279 \mathrm{~cm}^{-1}$, sendo a última mais
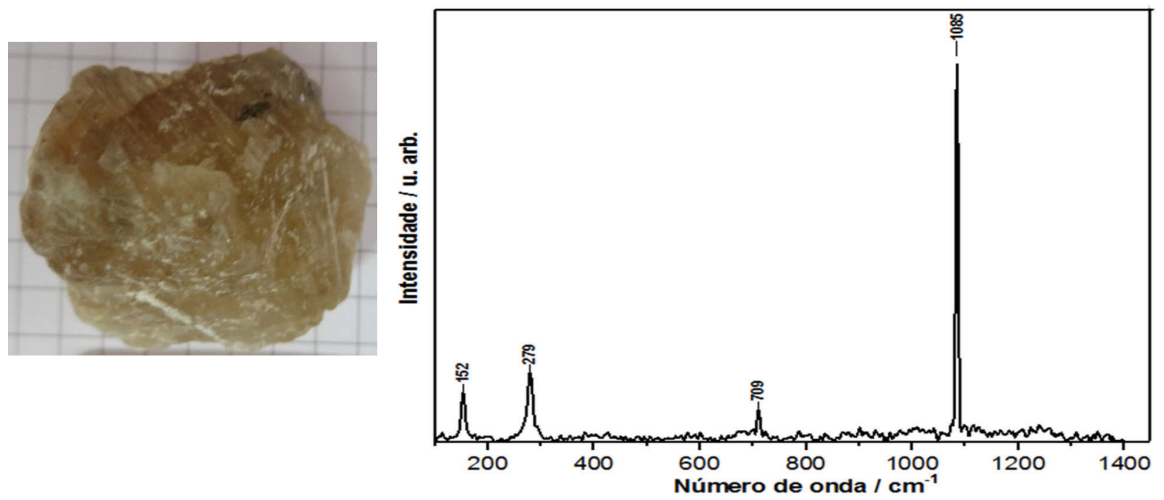

Figura 3. Amostra A170801 (calcita) e seu espectro Raman 
intensa que a primeira. Essas bandas estão relacionadas às vibrações externas do grupo $\mathrm{CO}_{3}{ }^{2-}$, que envolvem oscilações translacionais e rotatórias desse grupo. ${ }^{37}$

\section{Amostra A170802}

A análise por espectroscopia Raman da amostra A170802 permitiu caracterizá-la como sendo constituída pelo mineral espodumênio (Figura 4). O espodumênio possui a formula química $\mathrm{LiAlSi}_{2} \mathrm{O}_{6} \mathrm{e}$ pertence ao grupo dos piroxênios, fórmula geral $\mathrm{M} 2 \mathrm{M}_{1} \mathrm{Si}_{2} \mathrm{O}_{6}$, apresentando simetria monoclínica e grupo espacial $C 2 / c$ à baixa pressão. ${ }^{40} \mathrm{Na}$ estrutura desse mineral, o sítio $\mathrm{M} 1$ forma cadeias de $\mathrm{AlO}_{6}$ que separam as cadeias tetraédricas de $\mathrm{SiO}_{4}$ e o sítio M2 apresenta coordenação 6 , sendo este ocupado por $\mathrm{Li}^{41} \mathrm{O}$ espodumênio também apresenta três oxigênios em posições simetricamente não equivalentes: O1, O2 e O3. Os oxigênios O1 localizam-se no ápice do tetraedro de $\mathrm{SiO}_{4}$, os oxigênios $\mathrm{O} 2$ estão na base do tetraedro juntamente com os oxigênios $\mathrm{O}$, que são considerados oxigênios em ponte $\left(\mathrm{O}_{\mathrm{p}}\right)$ por estarem ligados a dois átomos de silício, enquanto que os oxigênios $\mathrm{O} 1$ e $\mathrm{O} 2$ não estão em ponte $\left(\mathrm{O}_{\mathrm{np}}\right)$, pois cada um está ligado a apenas um átomo de silício. ${ }^{42,43}$

O espectro Raman dos silicatos de cadeias simples, como o espodumênio, são caracterizados por quatro tipos de vibrações: as de estiramento dos oxigênios que não estão em ponte $\left(\mathrm{Si}-\mathrm{O}_{\mathrm{np}}\right)$, as de estiramento de oxigênios em ponte $\left(\mathrm{Si}-\mathrm{O}_{\mathrm{p}}\right)$, as vibrações de deformação da ligação O-Si-O e os modos de vibração cátion-oxigênio (M-O), que incluem os cátions M1 e M2.44

O espectro Raman obtido para a amostra A170802 é mostrado na Figura 5. As bandas correspondentes às vibrações $\mathrm{Si}-\mathrm{O}_{\mathrm{np}}$ são observadas em $1016 \mathrm{~cm}^{-1}, 1069 \mathrm{~cm}^{-1}$ e $1101 \mathrm{~cm}^{-1}$. Os modos de estiramento das ligações $\mathrm{Si}-\mathrm{O}_{\mathrm{p}}$ estão localizados em $703 \mathrm{~cm}^{-1}$ e $783 \mathrm{~cm}^{-1}$.

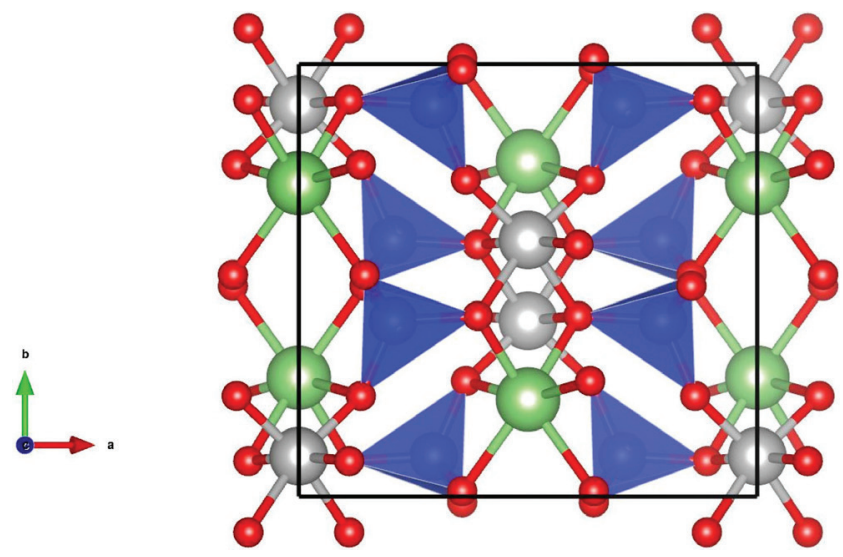

Figura 4. Estrutura cristalina do espodumênio, $\mathrm{LiAlSi}_{2} \mathrm{O}_{6}$ : tetraedros $\mathrm{Si}$ em azul, $\mathrm{Al}$ em cinza, Li em verde e $\mathrm{O}$ em vermelho ${ }^{45}$
A banda atribuída ao dobramento da ligação O-Si-O é observada em $519 \mathrm{~cm}^{-1}$ e a situada em $439 \mathrm{~cm}^{-1}$ corresponde ao estiramento da ligação Al-O pertencente ao grupo $\mathrm{AlO}_{6}$. As demais bandas presentes na região de baixa frequência do espectro são atribuídas às interações cátion-oxigênio. Esses dados foram comparados com a literatura e se encontram sumarizados na Tabela 3.

Tabela 3. Frequências Raman do mineral espodumênio $\left(\mathrm{cm}^{-1}\right)$

\begin{tabular}{ccc}
\hline Amostra A170802 & Ref. $^{42}$ & Ref. $^{43}$ \\
\hline--- & --- & 134 \\
--- & --- & 190 \\
248 & 249 & 254 \\
295 & 296 & 300 \\
335 & --- & --- \\
353 & 355 & 360 \\
390 & 393 & 397 \\
439 & 438 & 446 \\
519 & 522 & 523 \\
--- & 582 & 590 \\
703 & 705 & --- \\
783 & 783 & 712 \\
1016 & 1017 & --- \\
1069 & 1070 & 1076 \\
1101 & 1098 & -- \\
\hline & & \\
\hline & & \\
\hline
\end{tabular}

\section{Amostra A170803}

As análises de espectroscopia Raman da amostra A170803 possibilitaram a identificação de três minerais nesse espécime: a muscovita, o quartzo e a grafita.

A muscovita (Figura 6) é um mineral pertencente ao grupo das micas, cuja fórmula química é $\mathrm{KAl}_{2}\left(\mathrm{Si}_{3} \mathrm{Al}\right) \mathrm{O}_{10}(\mathrm{OH}, \mathrm{F})_{2} \cdot{ }^{46,47} \mathrm{Sua}$ estrutura é constituída por camadas tetraédricas-octaédricas-tetraédricas (TOT) unidas pelos cátions de $\mathrm{K}^{+}$, que ocupam os sítos octaédricos. O potássio é coordenado por dois íons $\mathrm{OH}^{-}$e por quatro oxigênios apicais que não estao em ponte $\left(\mathrm{O}_{\mathrm{np}}\right)$ e que pertencem às camadas tetraédricas superiores e inferiores. As camadas estruturais de muscovita possuem dois sítios tetraédricos, $\mathrm{T}_{1}$ e $\mathrm{T}_{2}$, que estão majoritariamente ocupados por $\mathrm{Si}^{4+}$. Tais camadas apresentam tetraedros individuais que estão unidos através do compartilhamento de oxigênios basais que se encontram em ponte $\left(\mathrm{O}_{\mathrm{p}}\right)$. Os cátions $\mathrm{Al}^{3+}$ formam uma camada de octaedros coordenados por quatro $\mathrm{O}_{\text {np }}$ e dois $\mathrm{OH}^{-} \cdot{ }^{48,49}$
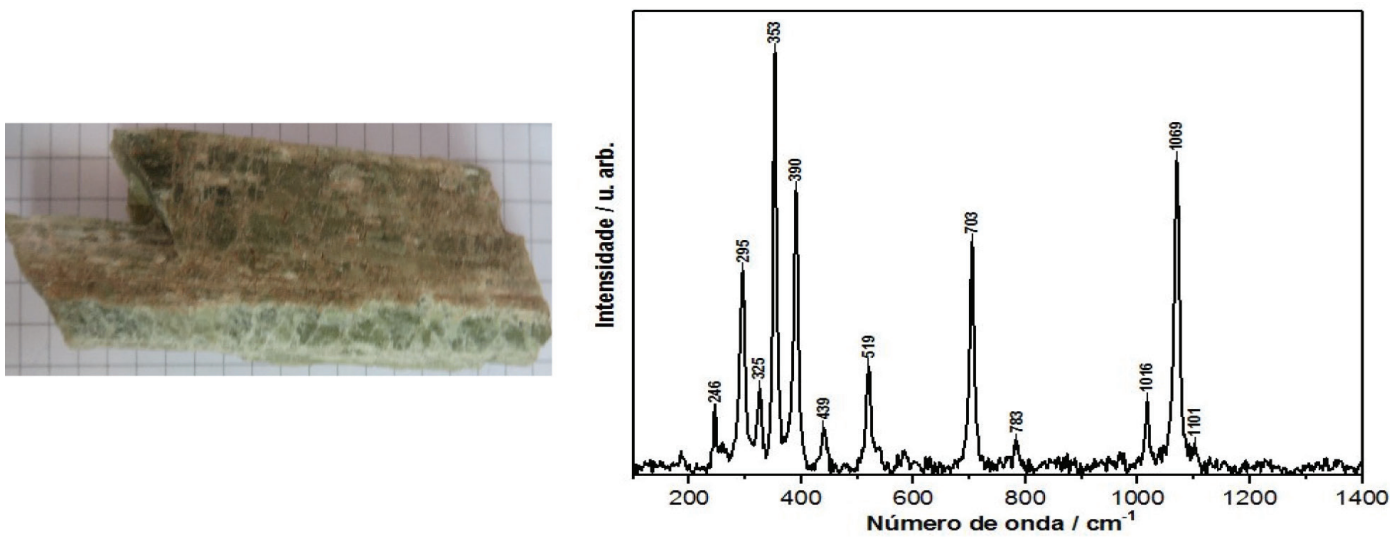

Figura 5. Amostra A170802 (espodumênio) e seu espectro Raman 


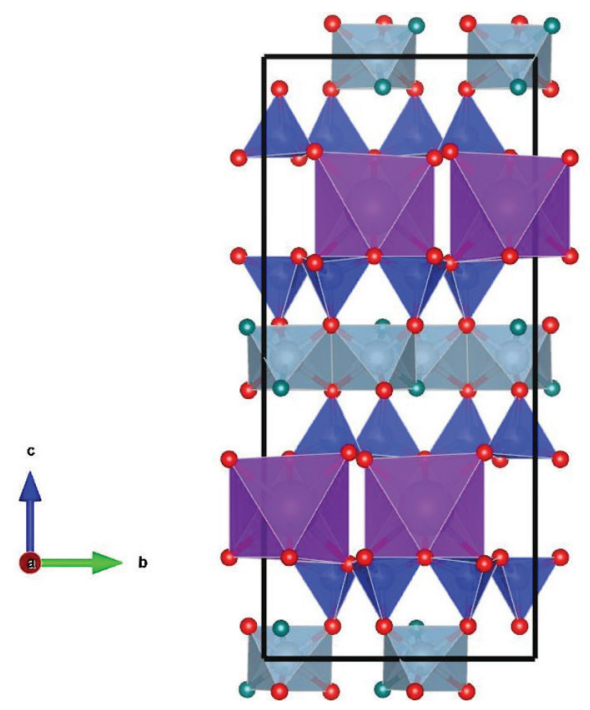

Figura 6. Estrutura cristalina da muscovita, $\mathrm{KAl}_{2}\left(\mathrm{Si}_{3} \mathrm{Al}\right) \mathrm{O}_{10}(\mathrm{OH}, \mathrm{F})_{2}$ : octaedros $\mathrm{K}$ em roxo, tetraedros $\mathrm{Si}$ em azul, octaedros $\mathrm{Al}$ em cinza, $\mathrm{O}$ em vermelho $e(\mathrm{OH}, \mathrm{F})$ em verde ${ }^{51}$
O espectro Raman obtido para a muscovita (Figura 7a) está de acordo com os dados da literatura e estes se encontram registrados na Tabela 4. As bandas localizadas nas frequências de $1092 \mathrm{~cm}^{-1} \mathrm{e}$ em $906 \mathrm{~cm}^{-1}$ são referentes às vibrações de estiramento de Si-O-Si e Si-O-Al, respectivamente. Já as bandas presentes em 747 e 701 cm-1, surgem em decorrência das vibrações de O-Al-O. ${ }^{48}$ As bandas localizadas em torno de 420 e $260 \mathrm{~cm}^{-1}$ envolvem vibração de tipos variados, sendo elas associadas principalmente às translações O-Al-O e O-Si-O. $\mathrm{O}$ pico Raman em $189 \mathrm{~cm}^{-1}$ pode ser atribuído ao estiramento das ligações $\mathrm{Al}-\mathrm{OH} .{ }^{50}$

O mineral quartzo (Figura 8) foi encontrado em uma pequena região da amostra A170803 após comparação do espectro obtido com a base de dados RRUFF. ${ }^{52} \mathrm{O}$ quartzo, $\mathrm{SiO}_{2}$, possui sistema hexagonal e grupo espacial $\mathrm{P} 3_{1} 21$ ou $\mathrm{P} 3_{2} 21$. Ele é um dos minerais mais abundantes da crosta terrestre, representando cerca de $12 \%$ de seu peso e podendo ser encontrado em rochas ígneas e sedimentares. ${ }^{53} \mathrm{O}$ espectro Raman obtido para esse mineral (Figura 7b) apresentou dez bandas relacionas a três tipos de vibrações, sendo elas o estiramento das ligações Si-O, a deformação angular de O-Si-O e a deformação angular de $\mathrm{Si}-\mathrm{O}-\mathrm{Si} .{ }^{54} \mathrm{As}$ informações obtidas foram comparadas e estão de acordo com dados presentes na literatura (Tabela 5).

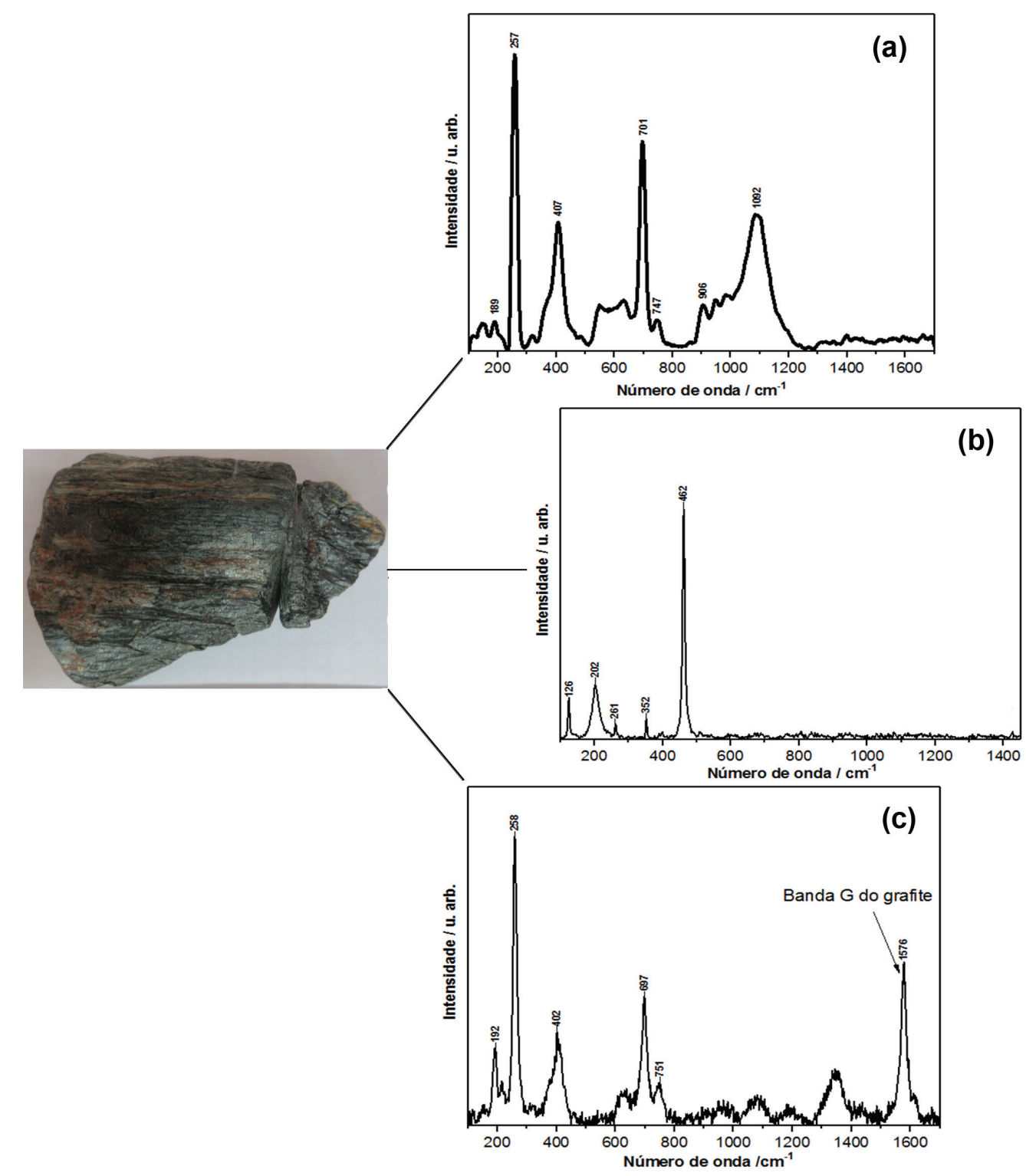

Figura 7. Amostra A170803 e os espectros Raman dos minerais que a constitui: espectro (a) muscovita, (b) quartzo e (c) mistura de grafita e muscovita 
Outro mineral encontrado na amostra A170803 foi a grafita (Figura 9). A grafita pertence ao sistema hexagonal, grupo espacial $\mathrm{P}_{3} / \mathrm{mmc}$ ou $\mathrm{R} \overline{3} \mathrm{~m}$ e é estruturalmente construída a partir do

Tabela 4. Frequências Raman do mineral muscovita $\left(\mathrm{cm}^{-1}\right)$

\begin{tabular}{cccc}
\hline Amostra A170803 & Ref. $^{50}$ & Ref. $^{48}$ & Ref. $^{48}$ \\
\hline 195 & 197 & 191 & 188 \\
264 & 263 & 262 & 262 \\
407 & 407 & 419 & 420 \\
--- & 579 & --- & --- \\
701 & 703 & 703 & 701 \\
750 & 755 & 752 & 753 \\
909 & 914 & 900 & 899 \\
1091 & 1127 & 1097 & 1102 \\
\hline
\end{tabular}

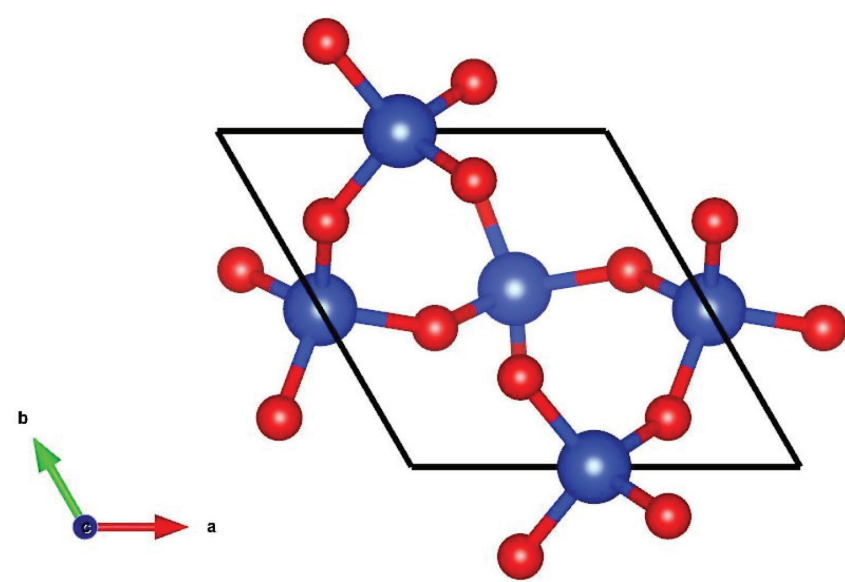

Figura 8. Estrutura cristalina do quartzo, $\mathrm{SiO}_{2}$ : Si em azul e O em vermelho ${ }^{55}$ empilhamento de planos hexagonais formados por átomos de carbono $\mathrm{sp}^{2}$. Os átomos de $\mathrm{C}$ presentes em um plano estão unidos por ligações covalentes, e esses planos se mantêm sobrepostos por meio de uma interação fraca de Van der Waals. ${ }^{59,60} \mathrm{O}$ espectro Raman da grafita é composto por uma única banda, conhecida como banda $\mathrm{G}$, que pode ser observada nas proximidades de $1580 \mathrm{~cm}^{-1},{ }^{1,62}$ sendo ela atribuída às vibrações longitudinais e transversais das ligações C-C no plano. ${ }^{63}$ No espectro obtido para a amostra A170803 (Figura 7c), a banda G foi localizada em $1579 \mathrm{~cm}^{-1}$, o que condiz com os dados presentes na literatura. ${ }^{59-63}$ Entretanto, não foi possível obter o espectro da grafita isoladamente, pois este mineral estava presente em grande quantidade na amostra juntamente com a muscovita. Deste modo, o espectro obtido possui bandas características da muscovita e a banda

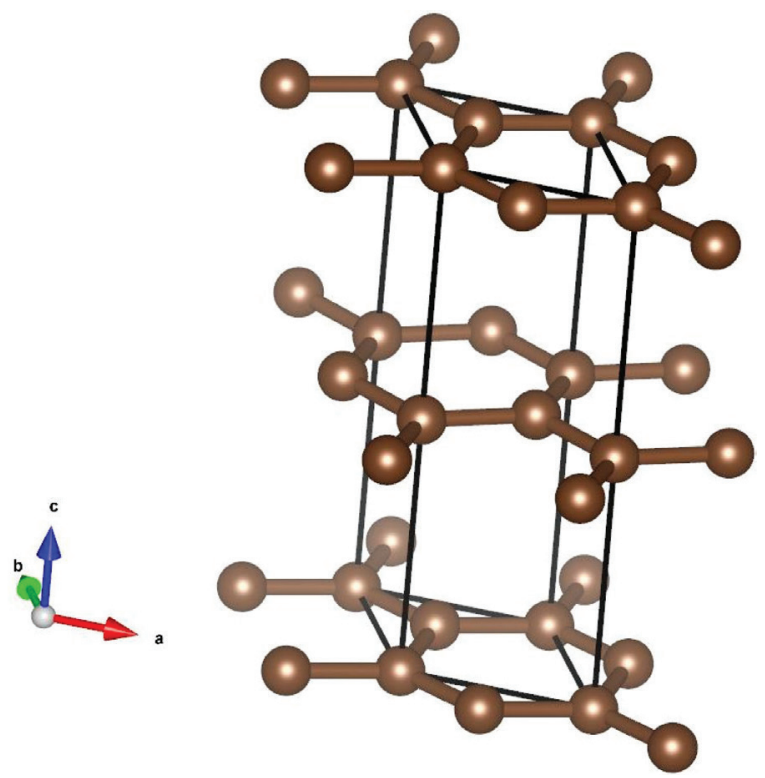

Figura 9. Estrutura cristalina da grafita, C. ${ }^{64}$

Tabela 5. Frequências Raman e tipos de vibrações do mineral quartzo $\left(\mathrm{cm}^{-1}\right)$

\begin{tabular}{|c|c|c|c|c|}
\hline Amostra A170803 & $\operatorname{Ref}^{56}$ & Ref. ${ }^{57}$ & $\operatorname{Ref}^{58}$ & Tipos de vibrações ${ }^{54}$ \\
\hline 126 & 128 & 128 & 127 & Deformação angular O-Si-O, deformação angular Si-O-Si e torção SI-O. \\
\hline 202 & 207.3 & 207.3 & 207 & Estiramento Si-O, Deformação angular O-Si-O e torção SI-O. \\
\hline 261 & 267 & 265.9 & 263 & Deformação angular O-Si-O e deformação angular Si-O-Si \\
\hline 352 & 358 & 356.5 & 354 & Deformação angular O-Si-O \\
\hline--- & 391 & 394.4 & 397 & Deformação angular O-Si-O \\
\hline--- & 403 & 403.9 & --- & ---- \\
\hline--- & 452 & --- & 453 & Deformação angular O-Si-O \\
\hline 462 & 466 & 466.4 & 465 & Estiramento Si-O e Deformação angular O-Si-O \\
\hline--- & 479 & --- & ----- & Deformação angular O-Si-O \\
\hline--- & 505 & --- & 501 & ---- \\
\hline--- & 695 & 696.8 & 694 & Estiramento $\mathrm{Si}-\mathrm{O}$ \\
\hline 795 & 795 & 796.9 & 794 & Estiramento $\mathrm{Si}-\mathrm{O}$ \\
\hline 807 & 806 & 809.3 & 805 & ---- \\
\hline--- & 1063 & 1063.1 & 1064 & Estiramento $\mathrm{Si}-\mathrm{O}$ \\
\hline 1082 & 1082 & 1085.2 & 1082 & Estiramento $\mathrm{Si}-\mathrm{O}$ \\
\hline --- & 1160 & 1160.2 & 1158 & Estiramento Si-O \\
\hline--- & 1228 & 1127 & 1228 & ---- \\
\hline
\end{tabular}


G da grafita, que foi identificada após comparação com a base de dados e com a literatura.

\section{CONCLUSÃO}

Os dados espectroscópicos coletados para as três amostras mineralógicas pertencentes à coleção de minerais do Departamento de Engenharia Civil da UFSCar permitiram obter informações necessárias para a correta caracterização dos seus constituintes, sendo este o objetivo deste trabalho. A comparação dos espectros Raman das amostras com os dados presentes na literatura possibilitou identificar que a amostra A170801 é formada pelo mineral calcita $\left(\mathrm{CaCO}_{3}\right)$, que é um dos polimorfos do carbonato de cálcio encontrados na natureza. Também foi possível inferir que a amostra A170802 é constituída por espodumênio, $\mathrm{LiAlSi}_{2} \mathrm{O}_{6}$, e que a amostra $\mathrm{A} 170803$ apresenta uma composição heterogênea, sendo formada pelos minerais grafita (C), quartzo $\left(\mathrm{SiO}_{2}\right)$ e muscovita $\left[\mathrm{KAl}_{2}\left(\mathrm{Si}_{3} \mathrm{Al}\right) \mathrm{O}_{10}(\mathrm{OH}, \mathrm{F})_{2}\right]$. É válido ressaltar que tais informações sobre $\mathrm{A} 170803$ não poderiam ter sido obtidas por meio da análise das propriedades comuns de caracterização de minerais como densidade, traço, dureza, brilho e clivagem; o que demostra o potencial da espectroscopia Raman para a caracterização de minerais. Ademais, as descobertas desse trabalho possibilitam o uso das três amostras corretamente identificadas em disciplinas e atividades acadêmicas, auxiliando a formação prática de novas gerações de profissionais qualificados em muitos campos da Ciência e Tecnologia.

\section{AGRADECIMENTOS}

Os autores agradecem à $\operatorname{Dr}^{\mathrm{a}}$ Marcilene Dantas Ferreira do Departamento de Engenharia Civil da Universidade Federal de São Carlos por ter cedido as amostras analisadas neste trabalho. Agradecem ainda à Fundação de Amparo à Pesquisa do Estado de São Paulo (FAPESP - processos 2017/15761-8 e 2013/03487-8) e ao CNPq (113844/2018-2) pelo suporte financeiro.

\section{REFERÊNCIAS}

1. Chalmin, E.; Menu, M; Vignaud, C.; Meas. Sci. Technol. 2003, 14, 1590.

2. De Faria, D. L. A.; Lopes, F.; Vib. Spectrosc. 2007, 45, 117.

3. Edwards, H.; Drummond, L.; Russ, J.; Spectrochim. Acta, A 1998, 54, 1849.

4. De Faria, D. L. A.; Lopes, F. N.; Souza, L. A. C.; Branco, H. D. O. C.; Quim. Nova 2011, 34, 1358.

5. Duffin, C. J.; Moody, R. T. J.; Gardner-Thorpe, C.; A History of Geology and Medicine, Geological Society: London, 2013.

6. Braga, P. F. A.; França, S. C. A. Em Valorização de Pegmatitos Litiníferos; Martins, L. M. P., Oliveira, D. P. S., Silva, R., Viegas, H. M. C., Bôas, R. C. V., eds; Fátima Engel: Lisboa, 2011, cap. 13.

7. Taoré, K.; Ouedraogo, G. V.; Blanchart, P.; Jernot, J.-P.; Gomina, M.; J. Eur. Ceram. Soc. 2007, 27, 1677.

8. Hazen, R. M.; J. Geol. Educ. 1984, 32, 288.

9. Callapez, P.; Brandão, J. M.; Livro de Actas do Congresso LusoBrasileiro de Historia das Ciências, Coimbra, Portugal, 2011.

10. Mazzoleni, P.; Barone, G; Raneri, S.; Aquilia, E.; Bersani, D.; Cirrincione, R.; Plinius 2016, 42, 112.

11. Barbosa, L. M.; Sitientibus 2000, 23, 9.

12. De Faria, D. L. A.; Afonso, M. C.; Edwards, H. G. M.; Revista do Museu de Arqueologia e Etnologia 2002, 12, 249.

13. Sala, O.; Fundamentos da Espectroscopia Raman e no Infravermelho, $2^{\text {nd }}$ ed., Editora Unesp: São Paulo, 2008.

14. Ando, R. A.; Dissertação de Mestrado, Universidade de São Paulo, Brasil, 2005.
15. De Faria, D. L. A.; Santos, L. G. C.; Gonçalves, N. S.; Quim. Nova 1997, $20,319$.

16. Sala, O.; Tese de Livre Docência, Universidade de São Paulo, Brasil, 1986.

17. Oliveira, L. D.; Cad. Tematicos Quim. Nova Esc. 2001, 4, 24.

18. Nasdala, L.; Smith, D.; Kaindl, R. Ziemann, M. A.; Em Spectroscopic methods in mineralogy, Beran, A., Libowitzky, E., eds.; Eötvös University Press: Budapest, 2004, cap. 7

19. Hope, G.; Woods, R.; Munce, C.; Miner. Eng. 2001, 14, 1565.

20. Das, S.; Hendry, M. J.; Chem. Geol. 2011, $290,101$.

21. Haskin, L. A.; Wang, A.; Rockow, K. M.; Jolliff, B. L.; Korotev, R. L.; Viskupic, K. M.; J. Geophys. Res.: Planets 1997, 102, 19293.

22. Andò, S.; Garzanti, E.; Geological Society, London, Special Publications 2014, 386, 395.

23. Bartholomew, P.; Dyar, M.; Brady, J.; J. Raman Spectrosc. 2015, 46, 889.

24. White, S. N.; Chem. Geol. 2009, 259, 240.

25. Vítek, P.; Ali, E. M.; Edwards, H. G.; Jehlička, J.; Cox, R.; Page, K.; Spectrochim. Acta, A 2012, 86, 320.

26. Griffith, W.; Nature 1969, 224, 264.

27. Rull, F.; Martinez-Frias, J.; Sansano, A.; Medina, J.; Edwards, H.; J. Raman Spectrosc. 2004, 35, 497.

28. Courreges-Lacoste, G. B.; Ahlers, B.; Pérez, F. R.; Spectrochim. Acta, A 2007, 68, 1023.

29. De Faria, D. L. A.; Santos, L.; Gonçalves, N.; Quim. Nova 1997, 20, 319.

30. Fredericci, C.; Ferreira, D. C.; Oliveira, M. C. B.; da Silveira, P. N.; Revista IPT: Tecnologia e Inovação 2016, 1, 13.

31. Roach, N.; Reddy, K.; Trends Soil Sci. 2004, 3, 1.

32. Sun, J.; Wu, Z.; Cheng, H.; Zhang, Z.; Frost, R. L.; Spectrochim. Acta, A 2014, 117, 158 .

33. Lippmann F.; Sedimentary Carbonate Minerals, $1^{\text {st }}$ ed., Springer-Verlag Berlin Heidelberg: Berlin, 1973.

34. Graf, D. L.; American Mineralogist: Journal of Earth and Planetary Materials 1990, 46, 1283.

35. Frost, R. L.; Hales, M. C.; Wain, D. L.; J. Raman Spectrosc. 2008, 39, 108.

36. Kontoyannis, C. G.; Vagenas; N. V.; Analyst (Cambridge, U. K.) 2000 , $125,251$.

37. Krishnamurti, D.; Proc. - Indian Acad. Sci., Math. Sci. 1957,46, 183

38. Herman, R. G.; Bogdan, C. E.; Sommer, A. J.; Simpson. D. R.; Appl. Spectrosc. 1987, 41, 437.

39. Rutt, H.; Nicola, J.; J. Phys. C: Solid State Phys. 1974, 7, 4522.

40. Pommier, C.; Denton, M. B.; Downs, R. T.; J. Raman Spectrosc. 2003, 34, 769 .

41. Wesełucha-Birczyńska A.; Słowakiewicz, M.; Natkaniec-Nowak, L.; Proniewicz, L.; Spectrochim. Acta, A 2011, 79, 789.

42. Cameron M.; Sueno, S.; Prewitt, C. T.; Papike, J. J.; Am. Mineral. 1973, $58,594$.

43. Clarck, J.; Appleman, D. E.; Papike, J. J.; Mineral. Soc. Am., Spec. Pap. 1969, 2, 31 .

44. Buzatu, A.; Buzgar, N.; An. Stiint. Univ. “Al. I. Cuza” Iasi, Sect. 2b: Geol. 2010, 56, 107.

44. Adams, D. M.; Hills, D. J.; Dalton Trans. 1977, 16, 1562.

45. Cameron, M.; Sueno, S.; Prewitt, C. T.; Papike, J. J.; American Mineralogist: Journal of Earth and Planetary Materials 1973, 58, 594.

46. Tlili, A.; Smith, D.; Beny, J.; Boyer, H.; Mineral. Mag. 1989, 53, 165.

47. Li, H.; Zhang, L.; Christy, A. G. Em Ultrahigh-Pressure Metamorphism: 25 years after the discovery of coesite and diamond; Dobrzhinetskaya, L., Faryad, S.W., Wallis, S., Cuthbert, S., eds.; Elsevier, 2011, cap. 7.

48. Šontevska, V.; Jovanovski, G.; Makreski, P.; Raškovska, A.; Šoptrajanova, B.; Acta Chim. Slov. 2008, 55, 757. 
49. Bárcena, J. L.; Urbina, M.; Rowlands, A. P.; Beneitez, P.; Millán, A.; Calderón, T.; Radiat. Prot. Dosim. 1999, 84, 289.

50. Singha, M.; Singh, L.; Indian J. Pure Appl. Phys. 2016, 54, 116.

51. Richardson, S. M.; Richardson, J. W.; Am. Mineral. 1982, 67, 69.

52. http://rruff.info/, acessada em abril 2019.

53. Saikia, B. J.; Parthasarathy, G.; Sarmah, N.; Bull. Mater. Sci. 2008, 31, 775 .

54. Etchepare, J.; Merian, M.; Smetankine, L.; J. Chem. Phys. 1974, 60, 1873.

55. Levien, L.; Prewitt, C. T.; Weidner, D. J.; Am. Mineral. 1980, 65, 920.
56. Saksena, B. D.; Proc. - Indian Acad. Sci., Math. Sci., A 1940, $12,93$.

57. Rasetti, F.; Il Nuovo Cimento 1932, 9, 72.

58. Nedungadi, T. M. K.; Indian Acad. Sci., Math. Sci. 1940, 11, 96.

59. Tuinstra, F.; Koenig, J. L.; J. Chem. Phys. 1970, 53, 1126.

60. Reich, S.; Thomsen, C.; Philos. Trans. R. Soc., A 2004, 362, 2271.

61. Ferrari, A. C.; Solid State Commun. 2007, 143, 47.

62. Hodkiewicz, J.; Characterizing carbon materials with Raman spectroscopy, Thermo Scientific Application Note. 2010, 51946.

63. Pimenta, M.; Dresselhaus, G.; Dresselhaus, M. S.; Cancado, L.; Jorio, A.; Saito, R.; Phys. Chem. Chem. Phys. 2007, 9, 1276.

64. Fayos, J.; J. Solid State Chem. 1999, 148, 278. 